\title{
MINOR ARC MOMENTS OF WEYL SUMS
}

\author{
M. P. HARVEY \\ Department of Mathematics, Royal Holloway, University of London, Egham, Surrey TW20 0EX, \\ United Kingdom \\ e-mail:michael.harvey@rhul.ac.uk
}

\begin{abstract}
(Received 29 July 2011; revised 10 January 2012; accepted 27 March 2012; first published online 2 August 2012)
\end{abstract}

\begin{abstract}
We obtain an improved bound for the $2^{k}$-th moment of a degree $k$ Weyl sum, restricted to a set of minor arcs, when $k$ is small. We then present some applications of this bound to some Diophantine problems, including a case of the Waring-Goldbach problem, and a particular family of Diophantine equations defined as the sum of a norm form and a diagonal form.
\end{abstract}

2010 Mathematics Subject Classification. 11L15, 11P55.

1. Introduction. The study of classical Weyl sums, those of the shape

$$
f(\alpha)=f_{k}(\alpha ; P):=\sum_{x \leq P} e\left(\alpha x^{k}\right), \quad e(x)=\exp (2 \pi i x),
$$

with $\alpha \in \mathbb{R}$, has many applications in analytic number theory. In particular, bounds for individual Weyl sums, as well as their mean values, have been conducive to a great deal of progress over the last century in Waring's problem and its generalisations via the Hardy-Littlewood method.

When $k$ is small, classical applications of the Hardy-Littlewood method resort to bounding an integral of the form

$$
\int_{\mathfrak{m}}\left|f_{k}(\alpha ; P)\right|^{s} \mathrm{~d} \alpha, \quad(\mathfrak{m} \subset[0,1))
$$

by applying Hua's inequality. This leads to the asymptotic formula for the number of representations of a large integer $n$ as the sum of $s k$-th powers, as soon as $s \geq 2^{k}+1$. The celebrated work of Vaughan $[12,13]$ attains an asymptotic formula for this problem with just $2^{k} k$-th powers, by saving a power of a logarithm over the main term for some suitable set of minor arcs $\mathfrak{m}$. While any logarithmic power saving will suffice for this problem, one might need to do better when considering Waring-Goldbach-type problems, or for studying the distribution of integer zeros of forms which split off diagonal forms.

We are interested in the possible $\lambda \in \mathbb{R}$ such that

$$
\int_{\mathfrak{m}}\left|f_{k}(\alpha ; P)\right|^{2^{k}} \mathrm{~d} \alpha \ll P^{2^{k}-k}(\log P)^{\varepsilon-\lambda}
$$

holds, for any $\varepsilon>0$, and for some suitable set of minor arcs $\mathfrak{m}$. For $k=3$, Vaughan's work [12] gives $\lambda=2-4 / \pi$. This was improved by Boklan [2], who showed that one can take $\lambda=3$. When $k \geq 4$, Vaughan [13] shows that $\lambda=2$ is acceptable, and remarks 
that one can replace this with $\lambda=(k-1)(k-2) / 2$. The goal of this current report is to improve on this value of $\lambda$, and to consider applications of this result to some Diophantine problems.

Let $\mathcal{G}_{i} \subset[1, P] \cap \mathbb{Z}\left(1 \leq i \leq 2^{k}\right)$ be a collection of sets, and define

$$
G_{i}(\alpha):=\sum_{x \in \mathcal{G}_{i}} e\left(\alpha x^{k}\right), \quad\left(1 \leq i \leq 2^{k}\right)
$$

for $k$ an integer satisfying $k \geq 4$. We establish the following.

TheOREM 1. Let $\mathfrak{m} \subset[0,1)$. Assume that for some $\eta \in \mathbb{R}$, we have

$$
\sup _{\alpha \in \mathfrak{m}}\left|G_{i}(\alpha)\right|^{2^{k-1}} \ll P^{2^{k-1}-1}(\log P)^{\eta}, \quad\left(1 \leq i \leq 2^{k-2}\right) .
$$

Then, for any $\varepsilon>0$,

$$
\int_{\mathfrak{m}} \prod_{i=1}^{2^{k}}\left|G_{i}(\alpha)\right| \mathrm{d} \alpha \ll P^{2^{k}-k}(\log P)^{\varepsilon-k(k-1) / 2} .
$$

We note that the logarithmic power saving is of the same strength of Boklan [2] when $k=3$. We also note that for $k \geq 6$, Boklan [3] derived an asymptotic formula for Waring's problem in $\frac{7}{8} 2^{k}$ variables by saving a power of a logarithm in the minor arc integral.

While we have stated the result with little restriction on the $\mathcal{G}_{i}$, in practice, one will require some conditions on the $\mathcal{G}_{i}$. They will need to be sufficiently dense in order for the major arc integral to dominate, as well as being well-distributed in order that the $G_{i}(\alpha)$ are well-approximated on the major arcs.

The proof of this theorem relies on a 'reduction' lemma, which relates the minor arc integral to one of exponential sums reduced to a thinner set, where we have better estimates. This argument is based on [2, Lemma D] and [13, Section 2], and is carried out in Section 2.

We start by mentioning some straightforward corollaries of Theorem 1 . Let $r_{s, k}(n)$ denote the number of representations of a large positive integer $n$ as the sum of $s k$-th powers of positive integers.

COROLlary 1 . Let $s=2^{k}$, for $k \geq 4$. Then we have, for any $\varepsilon>0$,

$$
r_{s, k}(n)=\mathfrak{S}_{s, k}(n) \frac{\Gamma\left((1+(1 / k))^{s}\right.}{\Gamma(s / k)} n^{(s / k)-1}+O\left(n^{(s / k)-1}(\log n)^{\varepsilon-k(k-1) / 2}\right) .
$$

This was established by Boklan [2, Corollary 1] when $k=3$. For $4 \leq k<6$, this improves on Vaughan [13], who proves this asymptotic formula with a weaker error term. As usual, $\mathfrak{S}_{s, k}(n)$ denotes the singular series, and satisfies $1 \ll \mathfrak{S}_{s, k}(n) \ll 1$.

An interesting variant of Waring's problem is to consider the number of representations of large integers as the sum of powers of primes. Kawada and Wooley [9] have shown that any large integer $n \equiv s(\bmod 240)$ is the sum of $s$ fourth powers of primes, provided that $s \geq 14$. Similarly, they showed that any large integer $n \equiv s(\bmod 2)$ is the sum of $s$ fifth powers of primes, provided that $s \geq 21$. They do not obtain asymptotic formulas for the number of such representations. 
Hua [6, Chapter VII] provides an asymptotic formula for the number of representations of a large number (under necessary congruence conditions) as the sum of $s k$-th powers of primes, provided that $s \geq 2^{k}+1$. We provide an asymptotic formula for $s=2^{k}$, but with fewer prime summands.

Let $R_{b, s-b, k}(n)$ denote the number of representations of $n$ as the sum of $s k$-th powers of positive integers, with at least $b$ of these being prime.

Corollary 2. Let $k \geq 4, s=2^{k}$ and $b=k(k-1) / 2-1$. Then, for any $\varepsilon>0$,

$$
R_{b, s-b, k}(n)=\mathfrak{S}_{k}(n) C n^{(s / k)-1}(\log n)^{-b}+O\left(n^{(s / k)-1}(\log n)^{\varepsilon-k(k-1) / 2}\right),
$$

where $C=C(k)$ is a positive constant,

$$
\mathfrak{S}_{k}(n)=\sum_{q=1}^{\infty} \sum_{\substack{a=1 \\(a, q)=1}}^{q} q^{-(s-b)} \phi(q)^{-b} S(q, a)^{s-b} S^{*}(q, a)^{b} e_{q}(-a n)
$$

$\phi(q)$ is Euler's totient function and

$$
S(q, a):=\sum_{r=1}^{q} e_{q}\left(a r^{k}\right), \quad S^{*}(q, a):=\sum_{\substack{r=1 \\(r, q)=1}}^{q} e_{q}\left(a r^{k}\right) .
$$

Our main application of Theorem 1 is to generalise the result of [4] to the case $k \geq 4$. Define a norm form to be a form

$$
N\left(x_{1}, \ldots, x_{k}\right):=N_{K / \mathbb{Q}}\left(x_{1} \omega_{1}+\cdots+x_{k} \omega_{k}\right),
$$

for $K / \mathbb{Q}$ a number field of degree $k$ with field norm $N_{K / \mathbb{Q}}$, and where $\left\{\omega_{1}, \ldots, \omega_{k}\right\}$ is an integral basis for $K$. It is clear that this is a form of degree $k$. Birch, Davenport and Lewis [1] exhibited an asymptotic formula for the number of integer zeros in an expanding region of the form

$$
N_{1}\left(x_{1}, \ldots, x_{k}\right)+N_{2}\left(y_{1}, \ldots, y_{k}\right)+z^{k}
$$

for degree $k$ norm forms $N_{1}, N_{2}$.

For non-zero integers $c, c_{1}, \ldots, c_{2^{k-1}}$, consider the form

$$
F:=c N\left(x_{1}, \ldots, x_{k}\right)+c_{1} y_{1}^{k}+\cdots+c_{2^{k-1}} y_{2^{k-1}}^{k} .
$$

Let $\mathscr{B}_{0} \subset \mathbb{R}^{k}, \mathscr{B}_{1}, \ldots, \mathscr{B}_{2^{k-1}} \subset \mathbb{R}$ be boxes, and define the box $\mathscr{B}:=\mathscr{B}_{0} \times \mathscr{B}_{1} \times \cdots \times$ $\mathscr{B}_{2^{k-1}}$. Let $P \mathscr{B}$ denote the set $\{P \mathbf{x}: \mathbf{x} \in \mathscr{B}\}$. Define the counting function

$$
\mathcal{N}(P):=\#\left\{(\mathbf{x}, \mathbf{y}) \in \mathbb{Z}^{k+2^{k-1}} \cap P \mathscr{B}: F=0\right\} .
$$

THEOREM 2. Let $\varepsilon>0$. We have

$$
\mathcal{N}(P)=\kappa P^{2^{k-1}}+O\left(P^{2^{k-1}}(\log P)^{\varepsilon-(k-1)(k-2) / 4}\right),
$$

for some $\kappa \geq 0$. Moreover, there exists $p_{0}>0$ such that if $F$ has non-singular $p$-adic zeros for each $p \leq p_{0}$ and a non-singular real zero, then we can choose $\mathscr{B}$ in such a way that $\kappa>0$. 
This implies the existence of non-trivial zeros of $F$ under the assumption of nonsingular local zeros. In other words, this establishes the clean Hasse principle for this family of forms. We remark in the case $k=4$, Vaughan's estimate [13] mentioned above is not strong enough to establish this result using our method of proof; the improvement made in Theorem 1 is essential. Coupled with Theorem 1, the key to Theorem 2 is to improve the bound of Birch, Davenport and Lewis [1, Lemma 1] for the mean square of a norm form exponential sum.

We first prove the reduction lemma in Section 2, before completing the proof of Theorem 1 in Section 3. We then briefly prove Corollaries 1 and 2 in Section 4. Finally, we prove Theorem 2 in Section 5 .

As usual, $\varepsilon$ will denote a small positive number that may change 'value' from one statement to the next. Throughout $k$ will always represent an integer with $k \geq 4$. All implied constants are allowed to depend on $k, K, c, c_{i}, \varepsilon$. We apply the usual notation that $e(z)=e^{2 \pi i z}, e_{q}(z)=e^{\frac{2 \pi i z}{q}}$.

The author would like to thank Dr. Tim Browning, Professor Trevor Wooley and Professor Jörg Brüdern for many useful conversations. This paper comprises part of the author's Ph.D. thesis at the University of Bristol.

2. Reduction Lemma. In this section, we prove the 'reduction' lemma that will facilitate our proof of Theorem 1. Our proof shall follow closely the work of Vaughan $[13$, Section 2]. We first prove the following useful result.

Lemma 2.1. Let $\mathcal{A} \subset[0,1]$ be measurable, and let $\mathcal{B}_{1}, \ldots, \mathcal{B}_{2^{j}} \subset[1, P] \cap \mathbb{Z}$ be a family of sets. Then for $3 \leq j \leq k$, we have

$$
\int_{\mathcal{A}} \prod_{i=1}^{2^{j}}\left|\sum_{x \in \mathcal{B}_{i}} e\left(\alpha x^{k}\right)\right| \mathrm{d} \alpha \ll P^{2^{j}-j} .
$$

Proof. On extending the range of integration and applying Hölder's inequality, we have

$$
\int_{\mathcal{A}} \prod_{i=1}^{2^{j}}\left|\sum_{x \in \mathcal{B}_{i}} e\left(\alpha x^{k}\right)\right| \mathrm{d} \alpha \leq \prod_{i=1}^{2^{j}}\left(\int_{0}^{1}\left|\sum_{x \in \mathcal{B}_{i}} e\left(\alpha x^{k}\right)\right|^{2^{j}} \mathrm{~d} \alpha\right)^{2^{-j}} .
$$

On considering the underlying Diophantine equations, for each $1 \leq i \leq 2^{j}$, we have

$$
\begin{aligned}
\int_{0}^{1}\left|\sum_{x \in \mathcal{B}_{i}} e\left(\alpha x^{k}\right)\right|^{2^{j}} \mathrm{~d} \alpha & \leq \int_{0}^{1}\left|\sum_{x \leq P} e\left(\alpha x^{k}\right)\right|^{2^{j}} \mathrm{~d} \alpha \\
& \ll P^{2^{j}-j},
\end{aligned}
$$

on using [13, Theorem 2] for $j=k$ and [13, Theorem B] for $3 \leq j<k$. The lemma now follows easily.

Define, for primes $p$ and a fixed $\tau$,

$$
\xi=\xi(\tau):=\left\{1 \leq n \leq P: p \mid n \Rightarrow p \notin\left((\log P)^{\tau}, P^{1 / 3 k}\right]\right\},
$$


and define

$$
\widehat{G}_{i}(\alpha):=\sum_{x \in \mathcal{G}_{i} \cap \xi} e\left(\alpha x^{k}\right)
$$

We now proceed to the reduction lemma. Recall the definitions above and (1.2).

Lemma 2.2. Let $\mathfrak{m} \subset[0,1)$. Assume that for some $\eta \in \mathbb{R}$, we have

$$
\sup _{\alpha \in \mathfrak{m}}\left|G_{i}(\alpha)\right|^{2^{k-1}} \ll P^{2^{k-1}-1}(\log P)^{\eta}, \quad\left(1 \leq i \leq 2^{k-2}\right) .
$$

Then for any fixed $\delta>0$, for sufficiently large $\tau$, we have

$$
\int_{\mathfrak{m}} \prod_{i=1}^{2^{k}}\left|G_{i}(\alpha)\right| \mathrm{d} \alpha \ll \prod_{i=2^{k}-1}^{2^{k}}\left(\int_{\mathfrak{m}}\left|\widehat{G}_{i}(\alpha)\right|^{2} \prod_{j=1}^{2^{k}-2}\left|G_{j}(\alpha)\right| \mathrm{d} \alpha\right)^{1 / 2}+P^{2^{k}-k}(\log P)^{-\delta} .
$$

Proof. On using the Cauchy-Schwarz inequality, we have

$$
\int_{\mathfrak{m}} \prod_{i=1}^{2^{k}}\left|G_{i}(\alpha)\right| \mathrm{d} \alpha \leq \prod_{i=2^{k}-1}^{2^{k}}\left(\int_{\mathfrak{m}}\left|G_{i}(\alpha)\right|^{2} \prod_{j=1}^{2^{k}-2}\left|G_{j}(\alpha)\right| \mathrm{d} \alpha\right)^{1 / 2} .
$$

Using Lemma 2.1, it suffices to show, for $i=2^{k}-1$ and $i=2^{k}$ that

$$
\int_{\mathfrak{m}}\left|G_{i}(\alpha)\right|^{2} \prod_{j=1}^{2^{k}-2}\left|G_{j}(\alpha)\right| \mathrm{d} \alpha \ll \int_{\mathfrak{m}}\left|\widehat{G}_{i}(\alpha)\right|^{2} \prod_{j=1}^{2^{k}-2}\left|G_{j}(\alpha)\right| \mathrm{d} \alpha+P^{2^{k}-k}(\log P)^{-2 \delta} .
$$

Assuming $i=2^{k}$, we shall partition $\mathcal{G}_{2^{k}} \times \mathcal{G}_{2^{k}}$ into the following disjoint sets:

$$
\begin{aligned}
\mathfrak{A} & :=\left\{(x, y) \in \mathcal{G}_{2^{k}} \times \mathcal{G}_{2^{k}}:(x, y)>(\log P)^{\tau}\right\}, \\
\mathfrak{B} & :=\left\{(x, y) \in \mathcal{G}_{2^{k}} \times \mathcal{G}_{2^{k}}:(x, y)<(\log P)^{\tau}, y \notin \xi\right\}, \\
\mathfrak{C} & :=\left\{(x, y) \in \mathcal{G}_{2^{k}} \times \mathcal{G}_{2^{k}}:(x, y)<(\log P)^{\tau}, x \notin \xi, y \in \xi\right\}, \\
\mathfrak{D} & :=\left\{(x, y) \in \mathcal{G}_{2^{k}} \times \mathcal{G}_{2^{k}}:(x, y)<(\log P)^{\tau}, x \in \xi, y \in \xi\right\} .
\end{aligned}
$$

It is clear that

$$
\begin{aligned}
\left|G_{2^{k}}(\alpha)\right|^{2} & =\sum_{(x, y) \in \mathcal{G}_{2^{k}} \times \mathcal{G}_{2^{k}}} e\left(\alpha\left(x^{k}-y^{k}\right)\right) \\
& =\left(\sum_{(x, y) \in \mathfrak{A}}+\sum_{(x, y) \in \mathfrak{B}}+\sum_{(x, y) \in \mathfrak{C}}+\sum_{(x, y) \in \mathfrak{D}}\right) e\left(\alpha\left(x^{k}-y^{k}\right)\right),
\end{aligned}
$$

and so

$$
\int_{\mathfrak{m}}\left|G_{2^{k}}(\alpha)\right|^{2} \prod_{j=1}^{2^{k}-2}\left|G_{j}(\alpha)\right| \mathrm{d} \alpha=J(\mathfrak{A})+J(\mathfrak{B})+J(\mathfrak{C})+J(\mathfrak{D}),
$$


where

$$
J(\mathfrak{U}):=\int_{\mathfrak{m}} \sum_{(x, y) \in \mathfrak{U}} e\left(\alpha\left(x^{k}-y^{k}\right)\right) \prod_{j=1}^{2^{k}-2}\left|G_{j}(\alpha)\right| \mathrm{d} \alpha .
$$

We shall examine each, in turn, starting with $J(\mathfrak{A})$. We have

$$
J(\mathfrak{A}) \leq \sum_{d>(\log P)^{\tau}} \int_{\mathfrak{m}}\left|\sum_{\substack{x, y \in \mathcal{G}_{2^{k}} \\(x, y)=d}} e\left(\alpha\left(x^{k}-y^{k}\right)\right)\right| \prod_{j=1}^{2^{k}-2}\left|G_{j}(\alpha)\right| \mathrm{d} \alpha
$$

After extending the range of integration and applying Hölder's inequality to the integral, this is bounded by

$$
\sum_{d>(\log P)^{\tau}}\left(\int_{0}^{1}\left|\sum_{\substack{x, y \in \mathcal{G}_{2^{k}} \\(x, y)=d}} e\left(\alpha\left(x^{k}-y^{k}\right)\right)\right|^{2^{k-1}} \mathrm{~d} \alpha\right)^{2^{1-k}} \prod_{j=1}^{2^{k}-2}\left(\int_{0}^{1}\left|G_{j}(\alpha)\right|^{2^{k}} \mathrm{~d} \alpha\right)^{2^{-k}}
$$

Applying Lemma 2.1, we find

$$
J(\mathfrak{A}) \ll P^{\left(2^{k}-k\right)\left(1-2^{1-k}\right)} \sum_{d>(\log P)^{\tau}}\left(\int_{0}^{1}\left|\sum_{\substack{x, y \in \mathcal{G}_{2 k} \\(x, y)=d}} e\left(\alpha\left(x^{k}-y^{k}\right)\right)\right|^{2^{k-1}} \mathrm{~d} \alpha\right)^{2^{1-k}} .
$$

The integral above is bounded by the number of solutions to

$$
x_{1}^{k}-y_{1}^{k}+\cdots+x_{2^{k-2}}^{k}-y_{2^{k-2}}^{k}=x_{2^{k-2}+1}^{k}-y_{2^{k-2}+1}^{k}+\cdots+x_{2^{k-1}}^{k}-y_{2^{k-1}}^{k},
$$

with each $x_{i}, y_{i} \leq P / d$. This is equal to

$$
\int_{0}^{1}\left|\sum_{x \leq P / d} e\left(\alpha x^{k}\right)\right|^{2^{k}} \mathrm{~d} \alpha \ll(P / d)^{2^{k}-k}
$$

by Lemma 2.1. Therefore,

$$
\begin{aligned}
J(\mathfrak{A}) & \ll P^{2^{k}-k} \sum_{d>(\log P)^{\tau}} d^{k 2^{1-k}-2} \\
& \ll P^{2^{k}-k}(\log P)^{-\tau\left(1-k 2^{1-k}\right)} .
\end{aligned}
$$

Taking $\tau \geq 2 \delta /\left(1-k 2^{1-k}\right)$ gives

$$
J(\mathfrak{A}) \ll P^{2^{k}-k}(\log P)^{-2 \delta} .
$$


We now move on to $J(\mathfrak{B})$. This is

$$
\begin{aligned}
& \leq \prod_{i=1}^{2^{k-2}} \sup _{\alpha \in \mathfrak{m}}\left|G_{i}(\alpha)\right| \int_{\mathfrak{m}} \sum_{(x, y) \in \mathfrak{B}} e\left(\alpha\left(x^{k}-y^{k}\right)\right)\left|\prod_{j=2^{k-2}+1}^{2^{k}-2}\right| G_{j}(\alpha) \mid \mathrm{d} \alpha \\
& \ll P^{\left(2^{k-1}-1\right) / 2}(\log P)^{\eta} \int_{\mathfrak{m}}\left|\sum_{(x, y) \in \mathfrak{B}} e\left(\alpha\left(x^{k}-y^{k}\right)\right)\right| \prod_{j=2^{k-2}+1}^{2^{k}-2}\left|G_{j}(\alpha)\right| \mathrm{d} \alpha,
\end{aligned}
$$

by the assumption of the lemma, and noting that the value of $\eta$ may change throughout this proof, but will always be a bounded constant depending only on at most $k$.

On extending the range of integration and applying Hölder's inequality, the integral above is bounded by

$$
\left(\int_{0}^{1}\left|\sum_{\alpha, y) \in \mathfrak{B}} e\left(\alpha\left(x^{k}-y^{k}\right)\right)\right|_{i=2^{k-2}+1}^{2}\left|G_{i}(\alpha)\right|^{2} \mathrm{~d} \alpha\right)^{1 / 2} \prod_{j=2^{k-1}-1}^{2^{k}-2}\left(\int_{0}^{1}\left|G_{j}(\alpha)\right|^{2^{k}} \mathrm{~d} \alpha\right)^{2^{-k}}
$$

On considering the underlying Diophantine equation, the first integral above can be majorised by replacing each $G_{i}(\alpha)$ with $f(\alpha)$, defined in (1.1). Hence, on using Lemma 2.1 for the remaining integrals above, we have

$$
J(\mathfrak{B}) \ll P^{\left(3\left(2^{k-1}\right)-k-1\right) / 2}(\log P)^{\eta}\left(\int_{0}^{1}\left|\sum_{(x, y) \in \mathfrak{B}} e\left(\alpha\left(x^{k}-y^{k}\right)\right)\right|^{2}|f(\alpha)|^{2^{k-1}-4} \mathrm{~d} \alpha\right)^{1 / 2} .
$$

The integral above is treated in [13, Section 2], and we can conclude,

$$
\begin{aligned}
J(\mathfrak{B}) & \ll P^{2^{k}-k}(\log P)^{\eta}(\log P)^{\varepsilon-\tau\left(1-2^{3-k}\right) /\left(2^{k-1}-2\right)} \\
& \leq P^{2^{k}-k}(\log P)^{-2 \delta}
\end{aligned}
$$

on taking $\tau>(2 \delta+\eta+\varepsilon)\left(2^{k-1}-2\right) /\left(1-2^{3-k}\right)$.

Clearly, $J(\mathfrak{C})$ can be treated in the same way as $J(\mathfrak{B})$ on interchanging $x$ and $y$. Moving onto the final piece $J(\mathfrak{D})$, we have

$$
\begin{aligned}
J(\mathfrak{D})= & \int_{\mathfrak{m}} \sum_{(x, y) \in \mathfrak{D}} e\left(\alpha\left(x^{k}-y^{k}\right)\right) \prod_{j=1}^{2^{k}-2}\left|G_{j}(\alpha)\right| \mathrm{d} \alpha \\
= & \int_{\mathfrak{m}}\left|\widehat{G_{2^{k}}}(\alpha)\right|^{2} \prod_{j=1}^{2^{k}-2}\left|G_{j}(\alpha)\right| \mathrm{d} \alpha \\
& -\sum_{d>(\log P)^{\tau}} \int_{\substack { \mathfrak{m} \\
\begin{subarray}{c}{x, y \in \mathcal{G}_{2^{k}} \cap \xi \\
(x, y)=d{ \mathfrak { m } \\
\begin{subarray} { c } { x , y \in \mathcal { G } _ { 2 ^ { k } } \cap \xi \\
( x , y ) = d } }\end{subarray}} e\left(\alpha\left(x^{k}-y^{k}\right)\right) \prod_{j=1}^{2^{k}-2}\left|G_{j}(\alpha)\right| \mathrm{d} \alpha .
\end{aligned}
$$


The latter term above, after extending the range of integration and applying Hölder's inequality, is bounded in absolute value by

$$
\sum_{d>(\log P)^{\tau}}\left(\int_{0}^{1}\left|\sum_{\substack{x, y \in \mathcal{G}_{2^{k}} \cap \xi \\(x, y)=d}} e\left(\alpha\left(x^{k}-y^{k}\right)\right)\right|^{2^{k-1}} \mathrm{~d} \alpha\right)^{2^{1-k}} \prod_{j=1}^{2^{k}-2}\left(\int_{0}^{1}\left|G_{j}(\alpha)\right|^{2^{k}} \mathrm{~d} \alpha\right)^{2^{-k}} .
$$

On considering the underlying equation, this is bounded by the expression in (2.1), and hence

$$
J(\mathfrak{D})=\int_{\mathfrak{m}}\left|\widehat{G_{2^{k}}}(\alpha)\right|^{2} \prod_{j=1}^{2^{k}-2}\left|G_{j}(\alpha)\right| \mathrm{d} \alpha+O\left(P^{2^{k}-k}(\log P)^{-2 \delta}\right),
$$

for $\tau \geq 2 \delta /\left(1-k 2^{1-k}\right)$.

The proof is complete on taking

$$
\tau \geq \max \left\{2 \delta /\left(1-k 2^{1-k}\right),(2 \delta+\eta+\varepsilon)\left(2^{k-1}-2\right) /\left(1-2^{3-k}\right)\right\} .
$$

3. Proof of Theorem 1. We can now use Lemma 2.2 to prove Theorem 1 . We require the following lemma.

Lemma 3.1. Let $\mathcal{A} \subset[0,1]$ be measurable, and let $\mathcal{B} \subset[1, P] \cap \mathbb{Z}$. Then

$$
\int_{\mathcal{A}}\left|\sum_{x \in \mathcal{B} \cap \xi} e\left(\alpha x^{k}\right)\right|^{2^{k}} \mathrm{~d} \alpha \ll P^{2^{k}-k}(\log P)^{\varepsilon-k(k-1) / 2} .
$$

Proof. On extending the range of integration, and comparing the underlying Diophantine equations, we have

$$
\int_{\mathcal{A}}\left|\sum_{x \in \mathcal{B} \cap \xi} e\left(\alpha x^{k}\right)\right|^{2^{k}} \mathrm{~d} \alpha \leq \int_{0}^{1}|\widehat{F}(\alpha)|^{2^{k}} \mathrm{~d} \alpha
$$

where

$$
\widehat{F}(\alpha):=\sum_{x \in \xi} e\left(\alpha x^{k}\right) .
$$

The lemma can then be evinced from [13, Section 2].

We now proceed to prove Theorem 1 .

Proof of Theorem 1. On applying Hölder's inequality, we have

$$
\int_{\mathfrak{m}} \prod_{i=1}^{2^{k}}\left|G_{i}(\alpha)\right| \mathrm{d} \alpha \ll \prod_{j=2^{k-2}+1}^{2^{k}} I_{j}^{1 / 3\left(2^{k-2}\right)}
$$


where

$$
I_{j}:=\int_{\mathfrak{m}}\left|G_{j}(\alpha)\right|^{3\left(2^{k-2}\right)} \prod_{i=1}^{2^{k-2}}\left|G_{i}(\alpha)\right| \mathrm{d} \alpha
$$

Hence, it suffices to prove, for each $2^{k-2}+1 \leq j \leq 2^{k}$, that

$$
I_{j} \ll P^{2^{k}-k}(\log P)^{\varepsilon-k(k-1) / 2} .
$$

We can apply Lemma 2.2 to $I_{j}$, on noting that we assume in Theorem 1 the hypothesis of Lemma 2.2. We obtain, for any $\delta>0$,

$$
I_{j} \ll \int_{\mathfrak{m}}\left|\widehat{G}_{j}(\alpha)\right|^{2}\left|G_{j}(\alpha)\right|^{3\left(2^{k-2}\right)-2} \prod_{i=1}^{2^{k-2}}\left|G_{i}(\alpha)\right| \mathrm{d} \alpha+P^{2^{k}-k}(\log P)^{-\delta}
$$

By Hölder's inequality, we have

$$
I_{j} \ll I_{j}^{1-1 / 3\left(2^{k-2}\right)}\left(\int_{\mathfrak{m}}\left|\widehat{G}_{j}(\alpha)\right|^{3\left(2^{k-2}\right)} \prod_{i=1}^{2^{k-2}}\left|G_{i}(\alpha)\right| \mathrm{d} \alpha\right)^{1 / 3\left(2^{k-2}\right)}+P^{2^{k}-k}(\log P)^{-\delta},
$$

hence

$$
I_{j} \ll \int_{\mathfrak{m}}\left|\widehat{G}_{j}(\alpha)\right|^{3\left(2^{k-2}\right)} \prod_{i=1}^{2^{k-2}}\left|G_{i}(\alpha)\right| \mathrm{d} \alpha+P^{2^{k}-k}(\log P)^{-\delta} .
$$

Applying Hölder's inequality to the integral above, we have

$$
\begin{aligned}
I_{j} & \ll\left(\int_{\mathfrak{m}}\left|\widehat{G}_{j}(\alpha)\right|^{2^{k}} \mathrm{~d} \alpha\right)^{3 / 4} \prod_{i=1}^{2^{k-2}}\left(\int_{\mathfrak{m}}\left|G_{i}(\alpha)\right|^{2^{k}} \mathrm{~d} \alpha\right)^{2^{-k}}+P^{2^{k}-k}(\log P)^{-\delta} \\
& \ll P^{3\left(2^{k}-k\right) / 4}(\log P)^{\varepsilon-3 k(k-1) / 8} \prod_{i=1}^{2^{k-2}} L_{i}^{2^{-k}}+P^{2^{k}-k}(\log P)^{-\delta},
\end{aligned}
$$

on using Lemma 3.1, and where

$$
L_{i}:=\int_{\mathfrak{m}}\left|G_{i}(\alpha)\right|^{2^{k}} \mathrm{~d} \alpha, \quad\left(1 \leq i \leq 2^{k-2}\right) .
$$

Applying Lemma 2.2 to $L_{i}$ and then using Hölder's inequality, we see that

$$
\begin{aligned}
L_{i} & \ll \int_{\mathfrak{m}}\left|\widehat{G}_{i}(\alpha)\right|^{2}\left|G_{i}(\alpha)\right|^{2^{k}-2} \mathrm{~d} \alpha+P^{2^{k}-k}(\log P)^{-\delta} \\
& \ll\left(\int_{\mathfrak{m}}\left|\widehat{G}_{i}(\alpha)\right|^{2^{k}} \mathrm{~d} \alpha\right)^{2^{1-k}} L_{i}^{1-2^{1-k}}+P^{2^{k}-k}(\log P)^{-\delta} .
\end{aligned}
$$


Hence,

$$
\begin{aligned}
L_{i} & \ll \int_{\mathfrak{m}}\left|\widehat{G}_{i}(\alpha)\right|^{2^{k}} \mathrm{~d} \alpha+P^{2^{k}-k}(\log P)^{-\delta} \\
& \ll P^{2^{k}-k}(\log P)^{\varepsilon-k(k-1) / 2}
\end{aligned}
$$

follows from Lemma 3.1. Combining this with (3.2), we obtain (3.1) and the proof is complete.

4. Proof of Corollaries 1 and 2. For the proof of Corollary 1, we apply Theorem 1 with each $G_{i}(\alpha)$ equal to $f(\alpha)$, defined in (1.1), and let $P=n^{1 / k}$. The assumption of the theorem is satisfied by Vaughan [13, Lemma 3] for the set of minor arcs defined in that lemma. The proof is then completed by using Theorem 1 and following the proof of Vaughan [13, Theorem 1]

In Corollary 2 , we shall apply Theorem 1 on setting $G_{1}(\alpha), \ldots, G_{b}(\alpha)$ to equal $g(\alpha)$, where

$$
g(\alpha):=\sum_{\substack{x \leq P \\ x \text { is prime }}} e\left(\alpha x^{k}\right)
$$

and setting $G_{b+1}(\alpha), \ldots, G_{s}(\alpha)$ to equal $f(\alpha)$. Recall that $s=2^{k}$ and $b=k(k-1) / 2-1$. Setting $P=n^{1 / k}$, it is clear that

$$
R_{b, s-b, k}(n)=\int_{0}^{1} g(\alpha)^{b} f(\alpha)^{s-b} e(-n \alpha) \mathrm{d} \alpha .
$$

For $2 \leq W \leq P$, define $\mathfrak{M}(W)$ to be the disjoint union of the intervals

$$
\mathfrak{M}_{a, q}(W):=\left\{\alpha \in[0,1):|q \alpha-a| \leq W P^{-k}\right\}
$$

over all coprime integers $a, q$ with $0 \leq a \leq q \leq W$. We now partition the unit interval as follows. Let $\mathfrak{M}:=\mathfrak{M}\left((\log P)^{\Delta}\right), \mathfrak{m}:=[0,1) \backslash \mathfrak{M}\left(P^{k 2^{1-k}}\right)$, and $\mathfrak{n}:=\mathfrak{M}\left(P^{k 2^{1-k}}\right) \backslash$ $\mathfrak{M}\left((\log P)^{\Delta}\right)$, where

$$
\Delta:=\frac{k^{2}(k-1) s}{2(s-b)}
$$

It then follows that

$$
R_{b, s-b, k}(n)=\left(\int_{\mathfrak{M}}+\int_{\mathfrak{m}}+\int_{\mathfrak{n}}\right) g(\alpha)^{b} f(\alpha)^{s-b} e(-n \alpha) \mathrm{d} \alpha .
$$

By Vaughan [13, Lemma 3], and noting that $s-b \geq 2^{k-2}$, we can apply Theorem 1 to show

$$
\int_{\mathfrak{m}} g(\alpha)^{b} f(\alpha)^{s-b} e(-n \alpha) \mathrm{d} \alpha \ll P^{s-k}(\log P)^{\varepsilon-k(k-1) / 2}=O\left(n^{(s / k)-1}(\log n)^{\varepsilon-k(k-1) / 2}\right) .
$$


Moving onto the integral over $\mathfrak{n}$, we apply Hölder's inequality to see that

$$
\begin{aligned}
\int_{\mathfrak{n}} g(\alpha)^{b} f(\alpha)^{s-b} e(-n \alpha) \mathrm{d} \alpha & \leq\left(\int_{\mathfrak{n}}|g(\alpha)|^{s} \mathrm{~d} \alpha\right)^{b / s}\left(\int_{\mathfrak{n}}|f(\alpha)|^{s} \mathrm{~d} \alpha\right)^{(s-b) / s} \\
& \ll P^{(s-k) b / s}\left(\int_{\mathfrak{n}}|f(\alpha)|^{s} \mathrm{~d} \alpha\right)^{(s-b) / s}
\end{aligned}
$$

on applying Lemma 2.1. It follows from Vaughan [14, Lemma 5.1] that

$$
\int_{\mathfrak{n}}|f(\alpha)|^{s} \mathrm{~d} \alpha \ll P^{s-k}(\log P)^{\varepsilon-\Delta / k} .
$$

Combining this with (4.3) and (4.2), we have

$$
\int_{\mathfrak{n}} g(\alpha)^{b} f(\alpha)^{s-b} e(-n \alpha) \mathrm{d} \alpha \ll P^{s-k}(\log P)^{\varepsilon-k(k-1) / 2}=O\left(n^{(s / k)-1}(\log n)^{\varepsilon-k(k-1) / 2}\right) .
$$

It remains to consider the integral over the major arcs $\mathfrak{M}$. Let

$$
v^{*}(\beta):=\sum_{2 \leq u \leq P^{k}} u^{1-1 / k}(\log u)^{-1} e(\beta u), \quad v(\beta):=k^{-1} \sum_{1 \leq u \leq P^{k}} u^{1-1 / k} e(\beta u),
$$

and recall the definitions of $S(q, a)$ and $S^{*}(q, a)$ from (1.3). Define

$$
\begin{aligned}
V^{*}(\alpha, q, a) & :=\phi(q)^{-1} S^{*}(q, a) v^{*}\left(\alpha-\frac{a}{q}\right), \\
V(\alpha, q, a) & :=q^{-1} S(q, a) v\left(\alpha-\frac{a}{q}\right) .
\end{aligned}
$$

It follows from Vaughan [15, Theorem 4.1] that

$$
f(\alpha)=V(\alpha, q, a)+O\left(q^{1 / 2+\varepsilon}\right),
$$

whenever $\alpha \in \mathfrak{M}_{a, q}\left((\log P)^{\Delta}\right)$. Hua [5, Lemma 6] uses the Siegel-Walfisz Theorem to show that

$$
g(\alpha)=V^{*}(\alpha, q, a)+O\left(P e^{-c \sqrt{\log P}}\right)
$$

for some constant $c>0$, whenever $\alpha \in \mathfrak{M}_{a, q}\left((\log P)^{\Delta}\right)$. Using (4.4) and (4.5), we have

$$
\begin{aligned}
\int_{\mathfrak{M}} g(\alpha)^{b} f(\alpha)^{s-b} e(-n \alpha) \mathrm{d} \alpha= & \sum_{q \leq W} \sum_{\substack{a=1 \\
(a, q)=1}} \int_{\mathfrak{M}_{a, q}\left((\log P)^{\Delta}\right)} V^{*}(\alpha, q, a)^{b} V(\alpha, q, a)^{s-b} e(-n \alpha) \mathrm{d} \alpha \\
& +O\left(n^{(s / k)-1}(\log n)^{-\delta}\right),
\end{aligned}
$$

for any $\delta>0$.

Combining all of the above, we have

$$
\begin{aligned}
R_{b, s-b, k}(n)= & \sum_{q \leq W} \sum_{\substack{a=1 \\
(a, q)=1}}^{q} q^{-(s-b)} \phi(q)^{-b} S(q, a)^{s-b} S^{*}(q, a)^{b} e_{q}(a n) \\
& \times \int_{-(\log P)^{\Delta} / q P^{k}}^{(\log P)^{\Delta} / q P^{k}} v(\beta)^{s-b} v^{*}(\beta)^{b} e(-n \beta) \mathrm{d} \beta+O\left(n^{(s / k)-1}(\log n)^{\varepsilon-k(k-1) / 2}\right) .
\end{aligned}
$$


Corollary 2 now follows on extending the ranges of $q$ and $\beta$ to infinity, and using standard estimates for $v(\beta)$ and $v^{*}(\beta)$ (see Vaughan [15, Chapter 2], for example).

5. Proof of Theorem 2. We prove Theorem 2 via the Hardy-Littlewood method. On top of Theorem 1, we shall need to improve on an estimate of Birch, Davenport and Lewis [1, Lemma 1] for the second moment of a norm form exponential sum. We first set up the Hardy-Littlewood machinery, before proving this result.

Let $c, c_{1}, \ldots, c_{2^{k-1}}$ be a collection of non-zero integers and $\mathscr{B}_{0} \subset \mathbb{R}^{k}$, $\mathscr{B}_{1}, \ldots, \mathscr{B}_{2^{k-1}} \subset \mathbb{R}$ be boxes. Define

$$
S_{i}(\alpha):=\sum_{x \in P \mathscr{B}_{i}} e\left(\alpha c_{i} x^{k}\right), \quad\left(1 \leq i \leq 2^{k-1}\right),
$$

and define

$$
T(\alpha):=\sum_{\mathbf{x} \in P \mathscr{B}_{0}} e(\alpha c N(\mathbf{x})) .
$$

Recalling (1.5) and using orthogonality, we have

$$
\mathcal{N}(P)=\int_{0}^{1} T(\alpha) S_{1}(\alpha) \cdots S_{2^{k-1}}(\alpha) \mathrm{d} \alpha .
$$

Recall the notation (4.1), and define $\mathfrak{M}:=\mathfrak{M}\left(P^{k 2^{1-k}}\right), \mathfrak{m}:=[0,1) \backslash \mathfrak{M}$. It follows that

$$
\mathcal{N}(P)=\left(\int_{\mathfrak{M}}+\int_{\mathfrak{m}}\right) T(\alpha) S_{1}(\alpha) \cdots S_{2^{k-1}}(\alpha) \mathrm{d} \alpha .
$$

The major arc integral can be calculated using standard techniques (see $[\mathbf{4}$, Sections 4-5], for example) and one can see that

$$
\int_{\mathfrak{M}} T(\alpha) S_{1}(\alpha) \cdots S_{2^{k-1}}(\alpha) \mathrm{d} \alpha=\kappa P^{2^{k-1}}+O\left(P^{2^{k-1}-\delta}\right),
$$

for some $\delta>0$. Here, $\kappa \geq 0$ and is positive, provided that the form (1.4) has non-singular $p$-adic and real zeros, and the boxes $\mathscr{B}_{0}, \mathscr{B}_{1}, \ldots, \mathscr{B}_{2^{k-1}}$ are chosen appropriately.

For the minor arcs, we apply Hölder's inequality to show

$$
\int_{\mathfrak{m}} T(\alpha) S_{1}(\alpha) \cdots S_{2^{k-1}}(\alpha) \mathrm{d} \alpha \leq\left(\int_{0}^{1}|T(\alpha)|^{2} \mathrm{~d} \alpha\right)^{1 / 2} \max _{i}\left(\int_{\mathfrak{m}}\left|S_{i}(\alpha)\right|^{2^{k}} \mathrm{~d} \alpha\right)^{1 / 2},
$$

where we have extended the range of integration of the first integral on the right-hand side above. The second integral can be treated with the following lemma.

LEMMA 5.1. For each $1 \leq i \leq 2^{k-1}$, we have

$$
\int_{\mathfrak{m}}\left|S_{i}(\alpha)\right|^{2^{k}} \mathrm{~d} \alpha \ll P^{2^{k}-k}(\log P)^{\varepsilon-k(k-1) / 2} .
$$

Proof. This follows from Theorem 1 on applying Vaughan [13, Lemma 3], and by noting the proof of $[\mathbf{4}$, Corollary 3.2]. 
It remains to estimate the first integral on the right-hand side of (5.1). Birch, Davenport and Lewis [1] show that this integral is bounded by $\ll P^{k+\varepsilon}$. The remainder of this paper will be devoted to proving the following lemma, which completes the proof of Theorem 2 .

LEMMA 5.2. We have

$$
\int_{0}^{1}|T(\alpha)|^{2} \mathrm{~d} \alpha \ll P^{k}(\log P)^{k-1} .
$$

By orthogonality, we have

$$
\int_{0}^{1}|T(\alpha)|^{2} \mathrm{~d} \alpha \ll \sum_{n \leq t P^{k}} r(n)^{2},
$$

where $r(n)$ is the number of ideals of norm $n$ in $\mathcal{O}_{K}$, the ring of integers of our field $K$ from which our norm form $N$ is defined, and $t$ is a fixed constant. The proof of Lemma 5.2 then boils down to estimating this sum.

LEMMA 5.3. We have, for sufficiently large $x$,

$$
\sum_{n \leq x} r(n)^{2} \ll x(\log x)^{k-1} .
$$

In order to estimate $\sum_{n \leq x} r(n)^{2}$, we shall study the analytic properties of the Dirichlet series

$$
D(s):=\sum_{n=1}^{\infty} \frac{r(n)^{2}}{n^{s}},
$$

which converges for $\Re s>1$. Recall that the Dedekind zeta function of $K$ is defined to be

$$
\zeta_{K}(s):=\sum_{\mathfrak{a} \subset \mathcal{O}_{K}} \frac{1}{\mathcal{N}(\mathfrak{a})^{s}}=\sum_{n=1}^{\infty} \frac{r(n)}{n^{s}},
$$

where $\mathcal{N}(\mathfrak{a}):=\left[\mathcal{O}_{K}: \mathfrak{a}\right]$ is the norm of the ideal $\mathfrak{a}$. Our aim is to express $D(s)$ as a Rankin-Selberg convolution of the Dedekind zeta function with itself, via the use of Artin $L$-functions.

Let $E / F$ be a finite Galois extension of number fields, with Galois group $G=$ $\operatorname{Gal}(E / F)$. Let $\rho: G \rightarrow G L(n, \mathbb{C})$ be a continuous finite-dimensional representation of $G$, with corresponding character $\chi$. We define the Artin $L$-function to be

$$
L(s, \rho ; E / F)=L(s, \chi ; E / F):=\prod_{\mathfrak{p}} L_{\mathfrak{p}}(s, \rho, E / F)^{-1},
$$

where $\mathfrak{p}$ runs over all primes of $F$, and

$$
L_{\mathfrak{p}}(s, \rho, E / F):=\operatorname{det}\left(1-\rho\left(\mathrm{Fr}_{\mathfrak{p}}\right) N(\mathfrak{p})^{-s}\right),
$$


for unramified primes $\mathfrak{p}$ and a similar definition for ramified primes. Here, $\mathrm{Fr}_{\mathfrak{p}}$ denotes the Frobenius conjugacy class. A thorough exposition of Artin $L$-functions and the following properties can be found in Iwaniec and Kowalski [8, Section 5.13].

It is known that Artin $L$-functions have a meromorphic continuation to the entire complex plane and satisfy a functional equation. We also note that the Artin $L$-function attached to the trivial character of $G$, denoted by $1_{G}$, is

$$
L\left(s, 1_{G} ; E / F\right)=\zeta_{F}(s),
$$

the Dedekind zeta function of $F$. The following two properties of Artin $L$-functions will prove useful. Let $\chi_{1}, \chi_{2}$ be characters of $G$. Then we have

$$
L\left(s, \chi_{1}+\chi_{2} ; E / F\right)=L\left(s, \chi_{1} ; E / F\right) L\left(s, \chi_{2} ; E / F\right) .
$$

Let $E^{\prime}$ be an intermediate field of $E$ and $F$. Let $G=\operatorname{Gal}(E / F)$ and $H=\operatorname{Gal}\left(E / E^{\prime}\right)$. For $\psi$ a character of $H$, let $\psi^{G}$ denote the induced character of $G$. Then we have

$$
L\left(s, \psi, E / E^{\prime}\right)=L\left(s, \psi^{G}, E / F\right) .
$$

Recall our field $K$ is a finite extension of $\mathbb{Q}$ of degree $k$ and let $E$ be the Galois closure of $K$. Define $G=\operatorname{Gal}(E / \mathbb{Q})$ and $H=\operatorname{Gal}(E / K)$ and let $1_{H}$ denote the trivial character of $H$. It follows from (5.2) and (5.4) that

$$
\zeta_{K}(s)=L\left(s, 1_{H}, E / K\right)=L\left(s,\left(1_{H}\right)^{G}, E / \mathbb{Q}\right) .
$$

Let $\rho$ be the representation corresponding to the character $\left(1_{H}\right)^{G}$.

LEMMA 5.4. $D(s)$ can be meromorphically continued to the half plane $\Re s>1 / 2$, where it satisfies the following equation:

$$
D(s)=L(s, \rho \otimes \rho, E / \mathbb{Q}) f(s, \rho)^{-1},
$$

where

$$
f(s, \rho)=a \prod_{p} \Phi_{p}\left(p^{-s}\right)^{-1}
$$

for some non-zero constant $a \in \mathbb{C}$ depending on ramified primes, and $\Phi_{p}(t)$ is a polynomial defined by

$$
\Phi_{p}(t)=1+\sum_{m=2}^{k^{2}-1} b_{m} t^{m}
$$

for constants $b_{m}$ depending on $\rho$.

Proof. This follows on consulting Moroz [11, Theorem 1, p. 85].

We wish to factor $L(s, \rho \otimes \rho, E / \mathbb{Q})$ into a product of Artin $L$-functions attached to irreducible representations. By (5.3), we need to decompose the representation $\rho \otimes \rho$ of $G$ into irreducibles. Suppose that

$$
\rho \otimes \rho=\oplus_{i=1}^{r} n_{i} \rho_{i}
$$


where $n_{1}, \ldots, n_{r}$ are positive integers and $\rho_{1}, \ldots, \rho_{r}$ are irreducible representations of $G$. Since $\rho$ is a representation induced from a trivial representation, it follows from Isaacs [7, Theorem 5.18] that the trivial representation of $G$ must appear in the factorisation of $\rho$, and hence appears in the factorisation of $\rho \otimes \rho$. Therefore, we may assume that $\rho_{1}$ is the trivial representation of $G$.

LEMMA 5.5. Recall the notation above. We have, for sufficiently large $x$,

$$
\sum_{n \leq x} r(n)^{2} \sim c x(\log x)^{n_{1}-1},
$$

for $c:=\lim _{s \rightarrow 1} D(s)(s-1)^{n_{1}} /\left(n_{1}-1\right)$ !.

Proof. By Lemma 5.4, (5.5) and (5.3), for $\Re s>1 / 2$, we have

$$
D(s)=f(s, \rho)^{-1} \prod_{i=1}^{r} L\left(s, \rho_{i}, E / \mathbb{Q}\right)^{n_{i}},
$$

and $f(s, \rho)$ is absolutely convergent in this region. We have $L\left(s, \rho_{1}, E / \mathbb{Q}\right)^{n_{1}}=\zeta(s)^{n_{1}}$ which has a pole of order $n_{1}$ at $s=1$. For $2 \leq i \leq r$, we have $\rho_{i}$ is a non-trivial irreducible representation, and it follows from Iwaniec and Kowalski [8, Corollary 5.47] that $L\left(s, \rho_{i}, E / \mathbb{Q}\right)$ has no pole at $s=1$. Therefore, $D(s)$ has a pole of order $n_{1}$ at $s=1$. An application of the Wiener-Ikehara theorem (see Montgomery and Vaughan [10, Section 8.3], for example) completes the proof.

To complete the proof of Lemma 5.3, it remains to prove the following.

LEMMA 5.6. The number of times that the trivial representation appears in the decomposition of $\rho \otimes \rho$ does not exceed $k$.

Proof. For two characters $\chi, \phi$ of a finite group $G$, define an inner product by

$$
\langle\chi, \phi\rangle=\langle\chi, \phi\rangle_{G}:=|G|^{-1} \sum_{g \in G} \chi(g) \overline{\phi(g)},
$$

where $\overline{\phi(g)}$ denotes the complex conjugate of the value of $\phi$ at $g$. This inner product is bilinear, and also has the following useful orthogonality property. Suppose $\chi$ and $\phi$ are irreducible characters of $G$, then

$$
\langle\chi, \phi\rangle=\left\{\begin{array}{l}
1, \text { if } \chi=\phi, \\
0, \text { otherwise }
\end{array}\right.
$$

It follows from bilinearity and the orthogonality property that $n_{1}=\left\langle\left(1_{H}\right)^{G}\left(1_{H}\right)^{G}, 1_{G}\right\rangle$. To prove the lemma, it suffices to check that

$$
\left\langle\left(1_{H}\right)^{G}\left(1_{H}\right)^{G}, 1_{G}\right\rangle \leq k .
$$

It is well known (see Isaacs [7, Theorem 5.18], for example) that,

$$
\left(1_{H}\right)^{G}=\sum_{i=1}^{t} m_{i} \phi_{i}
$$


where $m_{1}, \ldots, m_{t}$ are positive integers and $\phi_{1}, \ldots, \phi_{t}$ are irreducible characters of $G$ satisfying

$$
\sum_{i=1}^{t} m_{i} \phi_{i}(1)=k, \quad m_{i} \leq \phi_{i}(1) \quad(1 \leq i \leq t) .
$$

Therefore,

$$
\left(1_{H}\right)^{G}\left(1_{H}\right)^{G}=\sum_{i, j=1}^{t} m_{i} m_{j} \phi_{i} \phi_{j}
$$

By bilinearity,

$$
\begin{aligned}
\left\langle\left(1_{H}\right)^{G}\left(1_{H}\right)^{G}, 1_{G}\right\rangle & =\sum_{i, j=1}^{t} m_{i} m_{j}\left\langle\phi_{i} \phi_{j}, 1_{G}\right\rangle \\
& =\sum_{i, j=1}^{t} m_{i} m_{j}\left\langle\phi_{i}, \overline{\phi_{j}}\right\rangle
\end{aligned}
$$

with this last line clearly following from the definition (5.6). For each $1 \leq i \leq t$, there is at most one $j$ such that $\phi_{i}=\overline{\phi_{j}}$. Therefore, by the orthogonality relation (5.7), we have

$$
\sum_{i, j=1}^{t} m_{i} m_{j}\left\langle\phi_{i}, \overline{\phi_{j}}\right\rangle \leq \sum_{i=1}^{t} m_{i}^{2} \leq \sum_{i=1}^{t} m_{i} \phi_{i}(1)=k
$$

on using (5.8).

Lemma 5.3 now follows from Lemmas 5.5 and 5.6. Hence, the proof of Lemma 5.2 and thus the proof of Theorem 2 is complete.

ACKNOWLEDGEMENTS. This research was undertaken whilst the author was supported by an EPSRC Postgraduate studentship.

\section{REFERENCES}

1. B. J. Birch, H. Davenport and D. J. Lewis, The addition of norm forms, Mathematika 9 (1962), 75-82.

2. K. D. Boklan, A reduction technique in Waring's problem. I, Acta Arith. 65(2) (1993), $147-161$

3. K. D. Boklan, The asymptotic formula in Waring's problem, Mathematika 41(2) (1994), 329-347.

4. M. P. Harvey, On certain cubic forms in seven variables, Math. Proc. Cambridge Philos. Soc. 149(1) (2010), 21-47.

5. L. K. Hua, Some results in the additive prime-number theory, Q. J. Math. Oxford 9 (1938), 68-80.

6. L. K. Hua, Additive theory of prime numbers, in Translations of mathematical monographs, vol. 13 (Amer. Math. Soc., Providence, RI, 1965). 2006).

7. I. Isaacs, Character theory of finite groups (AMS Chelsea Publishing, Providence, RI,

8. H. Iwaniec and E. Kowalski, Analytic number theory (American Mathematical Society Colloquium Publications, Providence, RI, 2004). 
9. K. Kawada and T. D. Wooley, On the Waring-Goldbach problem for fourth and fifth powers, Proc. London Math. Soc. 83(1) (2001), 1-50.

10. H. L. Montgomery and R. C. Vaughan, Multiplicative number theory. I. Classical theory, in Cambridge studies in advanced mathematics, vol. 97 (Cambridge University Press, Cambridge, UK, 2007).

11. B. Z. Moroz, Analytic arithmetic in algebraic number fields, Lecture Notes in Mathematics, vol. 1205 (Springer-Verlag, Berlin, 1986).

12. R. C. Vaughan, On Waring's problem for cubes, J. Reine Angew. Math. 365 (1986), $122-170$.

13. R. C. Vaughan, On Waring's problem for smaller exponents. II, Mathematika 33(1) (1986), 6-22.

14. R. C. Vaughan, A new iterative method in Waring's problem, Acta Math 162(1-2) (1989), 1-71.

15. R. C. Vaughan, The Hardy-Littlewood method, second ed. (Cambridge University Press, Cambridge, UK, 1997). 